\title{
Nonlinear Adaptive Sensorless Speed Control of Induction Motors
}

\author{
Ren-Jye Chang ${ }^{1}$, Li-Chen Fu ${ }^{1,2}$
}

\author{
Department of Electrical Engineering ${ }^{1}$ \\ Department of Computer Science and Information Engineering ${ }^{2}$ \\ National Taiwan University \\ Taipei, Taiwan, Republic of China
}

\begin{abstract}
In this thesis, a nonlinear adaptive sensorless speed controiler for induction motors is proposed. In the controller, only the stator currents are assumed to be measurable. Flux observers and rotor speed estimator are designed to relax the need of flux and speed measurement. Besides, an estimator is also designed to overcome drifting problem of the rotor resistance.
\end{abstract}

\section{Nomenclature}

$\left\{V_{a}, V_{b}\right\}$ are stator voltages; $\left\{I_{a}, I_{b}\right\}$ are stator currents; $\left\{\psi_{a}, \psi_{b}\right\}$ are rotor fluxes; $\omega_{r}$ is the mechanical angular speed of the rotor; $R_{s}$ is the stator resistance; $R_{r}$ is the rotor resistance; $L_{s}$ is the stator self-inductance; $L_{r}$ is the rotor self-inductance; $M$ is the mutual inductance; $p$ is the number of pole-pairs; $J$ is the rotor inertia; $D$ is the damping coefficient; $T_{L}$ is the load torque; $k_{T}$ is the torque constant $\left(=\frac{3 p M}{2 L_{r}}\right) ; L_{o}$ equals to $\frac{L_{r}^{2}}{M}\left(L_{s}-\frac{M^{2}}{L_{r}}\right) ; \beta_{1}$ equals to $\frac{R_{s} L_{r}^{2}}{M} ; \beta_{2}$ equals to $p L_{r} ; \beta_{3}$ equals to $\frac{L_{r}^{2}}{M}$

\section{Introduction}

In early years of the induction motor control, all system states are assumed to be measurable and parameters are assumed to be known. Under these assumptions, classical field-oriented [1] and input-output. linearizing control technique [2] [3] are utilized to design the controller. Since in these schemes the flux sensors are required, this makes it impractical for implementation. Therefore flux observers are then designed to relax the need of flux measurement [4] [5]. Because observers proposed above are sensitive with respect to the rotor resistance, engineers then try to design estimators for the rotor resistance and/or other system parameters [6] [7]. After these researches, engineers nowadays try to design controllers and Hux observers which are adaptive with respect to both system parameters and/or the load. There are many researches published, such as [8] [16] [19] and [20]. All schemes above require speed measurement which violates the advantages of induction motors.Therefore, controllers that speed measurement are not required are preferable for practical irıplementation. The problem that to design a speed controller for induction motors without rotational transducers is then getting more and more popular and is usually called sensorless control problem. The firsi paper regarding sensorless control problem was presented in 1981 at a national conference in Japan. In this paper, vector control is utilized. After the presentation of this paper, many researches about sensorless vector control are proposed [12] [15]. In these researches, the analysis are mainly based on the steady state and proves are not discussed in detail. In all researches about sensorless control for induction motors, modern control theory such as robust, MRAS (Model Reference Adaptive System) is another popular trend. Here we follow this trend to design an adaptive sensorless speed controller for induction motors based on flux observer.

The layout of this paper is as follows. In section 1 , related researches are discussed briefly. In section 2 , the 
stationary $a-b$ reference frame model is introduced. In addition to derivation of the model, two lemmas which relates the speed tracking with the state tracking are preserted and proved. The main part of this thesis is section 3 in which observers and controller are explained and proved in detail. The experimental results are presented in section 4 . Finaliy, section 5 is the conclusion.

\section{Prelliminary and Problem For- mulation}

This sectior. is to briefly review the induction motor model and some useful properties and important basic results whicin facilitate the controller design.

\subsection{Motor Model}

If the induction motor never goes into the saturation region, and the air-gap $\mathrm{MMF}$ is sinusoidal, then it can be characterized by the following dynamic equations :

$$
\begin{aligned}
L_{o} \dot{I}_{a} & =-M R_{r} I_{a}-\beta_{1} I_{a}+R_{r} \psi_{a}+\beta_{2} \omega_{r} \psi_{b}+\beta_{3} V_{a} \\
L_{o} \dot{I}_{b} & =-M R_{r} I_{b}-\beta_{1} I_{b}-\beta_{2} \omega_{r} \psi_{a}+R_{r} \psi_{b}+\beta_{3} V_{b} \\
L_{r} \dot{\psi}_{a} & =-R_{r} \psi_{a}+M R_{r} I_{a}-\beta_{2} \omega_{r} \psi_{b} \\
L_{r} \dot{\psi}_{b} & =-R_{r} \psi_{b}+M R_{r} I_{b}+\beta_{2} \omega_{r} \psi_{a} \\
T_{e} & =k_{T}\left(\psi_{a} I_{b}-\psi_{b} I_{a}\right)
\end{aligned}
$$

where $L_{o}, \beta_{1}, \beta_{2}, \beta_{3}$ are constants defined in the nomenclature. Through some analysis based on Newton's law, the dynamics of the mechanical part is

$$
J \dot{\omega}_{r}+D \omega_{r}+T_{L}=T_{e}
$$

where $J>0$ is the rotor inertia, $D>0$ is the damping coefficient and $T_{L}$ is the load torque.

\subsection{Preliminaries}

The first lemma presented below establishes a relation between the speed tracking problem and the torque tracking problem.

\section{Lemma 1 .}

If the load torque $T_{L}$ can be written as

$$
T_{L}^{\prime}=\mu_{0} \cdot s\left(k, \omega_{r}\right)+\mu_{1} \omega_{r}+\mu_{2} \dot{\omega}_{r}
$$

with $\mu_{0}, \mu_{1}$ and $\mu_{2}$ being known positive constants and the sigmoidal function $s\left(k, \omega_{r}\right)$ be defined as :

$$
s\left(k, \omega_{r}\right)=\frac{2}{1+e^{-k \omega_{r}}}-1
$$

Then the speed tracking objective (i.e., $\omega_{r} \rightarrow \omega_{r d}$ ) can be achieved provided that the objective of torque tracking (i.e., $T_{e} \rightarrow T_{d}$ ) is achieved where the desired iorque is a smooth function given below :

$$
T_{d}=\left(J+\mu_{2}\right) \dot{\omega}_{r d}+\left(D+\mu_{1}\right) \omega_{r d}+\mu_{0} \cdot s\left(k, \omega_{r d}\right)
$$

\section{Proof :}

First, note that from (4), the desired torque is bounded if the desired speed is bounded. Since the sigmoidal function $\mathrm{s}(k, x)$ is

$$
\mathrm{s}(k, x)=\frac{2}{1+e^{-k \cdot x}}-1
$$

then its first-order derivative $\mathrm{g}(k, x)$ is

$$
\mathrm{g}(k, x)=\frac{2 k e^{-k \cdot x}}{\left(1+e^{-k \cdot x}\right)^{2}}
$$

It is obvious that $0 \leq \mathrm{g}(k, x) \leq \frac{k}{2}$. From the MeanValue theorem,

$$
\mathrm{s}\left(k, \omega_{r}\right)-\mathrm{s}\left(k, \omega_{r d}\right)=\left(\omega_{r}-\omega_{r d}\right) \cdot \frac{d \mathrm{~s}(k, x)}{d x}(k, \omega)
$$

where $\omega \in\left[\omega_{r}, \omega_{r d}\right]$, and hence

$T_{e}-T_{d}=\left(J+\mu_{2}\right) \dot{e}_{5}+\left(D+\mu_{1}+\mathrm{g}(k, \omega)\right) e_{5}, e_{5}=\omega_{r}-\omega_{r d}$

If $\left(T_{e}-T_{d}\right)$ approaches to 0 asymptotically, $e_{5}(t)$ approaches to 0 asymptotically, too. That is, $\omega_{r}$ approaches to $\omega_{r d}$ asymptotically.

The following lemma establishes a relation between the bound on the torque tracking error and the bound on the state tracking error.

\section{Lemma 2.}

If the desired torque $T_{d}$ can be expressed in the following form :

$$
T_{d}=k_{T}\left(\psi_{a d} I_{b d}-\psi_{b d} I_{a d}\right)
$$

where $I_{a d}\left(I_{b d}\right)$ and $\psi_{a d}\left(\psi_{b d}\right)$ are the desired values of the $a-b$ axis currents and fluxes, respectively. Then, the bound on the torque tracking error can be expressed as

$$
\left|T_{e}-T_{d}\right| \leq \frac{k_{T}}{2}\left(\|e\|^{2}+2\|e\|\left\|x_{d}\right\|\right)
$$

where $\boldsymbol{e}$ is the state tracking error vector and $\boldsymbol{x}_{\boldsymbol{d}}$ is the desired state vector respectively defined as

$x_{d}=\left[\begin{array}{c}x_{1 d} \\ x_{2 d} \\ x_{3 d} \\ x_{4 d}\end{array}\right]=\left[\begin{array}{c}I_{a d} \\ I_{b d} \\ \psi_{a d} \\ \psi_{b d}\end{array}\right], e=\left[\begin{array}{c}e_{1} \\ e_{2} \\ e_{3} \\ e_{4}\end{array}\right]=\left[\begin{array}{c}I_{a}-I_{a d} \\ I_{b}-I_{b d} \\ \psi_{a}-\psi_{a d} \\ \psi_{b}-\psi_{b d}\end{array}\right]$

\section{Proof :}

Refer to [9].

This lemma clearly leads to the following corollary. 


\section{Corollary 1.}

If the state tracking error converges to zero, i.e., $e \rightarrow$ $\mathrm{o}$, then the torque tracking error, $T_{e}-T_{d}$, converges to zero, too.

With the aid of these two lemmas, we see that the speed tracking can be achieved once the state tracking is achieved.

\subsection{Problem Formulation}

We try to design a speed controller for induction motors, which does not rely on any shaft sensor outputs and only measures the stator currents. All the parameters of the motor and of the load except the rotor resistance are assumed to be known. Given these information about the motor, for any speed command which is a secondorder continuously differentiable bounded function, this controller should be able to drive the motor to track the command.

\section{State Observer and Controller}

Before the thorough investigation on the observers and controllers, several assumptions will be presented below to make the problem more precise.

\section{Assumptions :}

(A1) All parameters of the motor and the load are known, except the rotor resistance.

(A2) The upper and the lower bounds of the rotor resistance are known and are denoted as $\bar{R}_{r}$ and $\underline{R}_{r}$, respectively.

(A3) The stator currents are measurable.

(A4) The load torque $T_{L}$ is a known function which satisfies that, if $T_{e}$ is bounded, the rotor speed $\omega_{r}$ is also bounded.

(A5) The desired rotor speed should be a bounded smooth function with known first and second order time derivatives.

(A6) The rotor speed is slowly varying and $\omega_{r d}-\delta_{\omega}<$ $\omega_{r}<\omega_{r d}+\delta_{\omega}$.

\section{Remark :}

It is obvious that $\omega_{r}$ is bounded once $T_{e}$ is assumed to be bounded. In other words, this kind of $T_{L}$ clearly satisfies assumption (A4).

Then we begin to introduce our observer and controller. For easy reference, define the notations for the observed values and the observation errors as follows:

$$
\begin{gathered}
\tilde{I}_{a}=I_{a}-\hat{I}_{a}, \tilde{I}_{b}=I_{b}-\hat{I}_{b}, \quad \tilde{\psi}_{a}=\psi_{a}-\hat{\psi}_{a} \\
\tilde{\psi}_{b}=\psi_{b}-\hat{\psi}_{b}, \quad \tilde{R}_{r}=R_{r}-\hat{R}_{r}, \quad \tilde{\omega}_{r}=\omega_{r}-\hat{\omega}_{r}
\end{gathered}
$$

where the symbol " denotes that it is an observed value and the symbol - denotes an observation error. The observers are derived as [8] :

$$
\begin{aligned}
L_{o} \dot{\hat{I}}_{a}= & k_{0} \tilde{I}_{a}-\left(M \hat{R}_{r}+\beta_{1}\right) I_{a}+\hat{R}_{r} \hat{\psi}_{a} \\
& +\beta_{2} \hat{\omega}_{r} \hat{\psi}_{b}+\beta_{3} V_{a}+u_{o 1}+u_{c 1} \\
L_{o} \dot{\hat{I}}_{b}= & k_{0} \tilde{I}_{b}-\left(M \hat{R}_{r}+\beta_{1}\right) I_{b}-\beta_{2} \hat{\omega}_{r} \hat{\psi}_{a} \\
& +\hat{R}_{r} \hat{\psi}_{b}+\beta_{3} V_{b}+u_{o 2}+u_{c 2} \\
L_{r} \dot{\hat{\psi}}_{a}= & -\hat{R}_{r} \hat{\psi}_{a}+M \hat{R}_{r} I_{a}-\beta_{2} \hat{\omega}_{r} \hat{\psi}_{b}+u_{o 3} \\
L_{r} \dot{\hat{\psi}}_{b}= & -\hat{R}_{r} \hat{\psi}_{b}+M \hat{R}_{r} I_{b}+\beta_{2} \hat{\omega}_{r} \hat{\psi}_{a}+u_{o 4}
\end{aligned}
$$

where the constant $k_{0}>0$ is a control gain.

Then design the following auxiliary signals as

$$
\eta_{a}=\tilde{Z}_{a}-\zeta_{a}, \quad \eta_{b}=\tilde{Z}_{b}-\zeta_{b}
$$

Define the state variables in a more compact form :

$$
\begin{gathered}
\mathrm{u}=\left[\begin{array}{l}
u_{1} \\
u_{2}
\end{array}\right]=\left[\begin{array}{l}
V_{a} \\
V_{b}
\end{array}\right], \mathrm{x}_{d}=\left[\begin{array}{l}
x_{1 d} \\
x_{2 d} \\
x_{3 d} \\
x_{4 d}
\end{array}\right]=\left[\begin{array}{c}
I_{a d} \\
I_{b d} \\
\psi_{a d} \\
\psi_{b d}
\end{array}\right] \\
\mathrm{x}=\left[\begin{array}{l}
x_{1} \\
x_{2} \\
x_{3} \\
x_{4}
\end{array}\right]=\left[\begin{array}{c}
I_{a} \\
I_{b} \\
\psi_{a} \\
\psi_{b}
\end{array}\right], \mathrm{e}=\left[\begin{array}{l}
e_{1} \\
e_{2} \\
e_{3} \\
e_{4}
\end{array}\right]=\left[\begin{array}{c}
I_{a}-I_{a d} \\
I_{b}-I_{b d} \\
\psi_{a}-\psi_{a d} \\
\psi_{b}-\psi_{b d}
\end{array}\right]
\end{gathered}
$$

then the state equations can be written concisely in a matrix form as follows:

$$
\dot{\mathrm{x}}=\left(\mathrm{A}_{0}+\mathrm{A}_{1}\right) x+\mathrm{Bu}
$$

where

$$
\begin{aligned}
\mathrm{A}_{0}= & {\left[\begin{array}{cccc}
-\left(\frac{M R_{r}}{L_{o}}+\frac{\beta_{1}}{L_{o}}\right) & 0 & 0 & 0 \\
0 & -\left(\frac{M R_{r}}{L_{o}}+\frac{\beta_{1}}{L_{o}}\right) & 0 & 0 \\
0 & 0 & -\frac{R_{r}}{L_{r}} & 0 \\
0 & 0 & 0 & -\frac{R_{r}}{L_{r}}
\end{array}\right] } \\
\mathrm{A}_{1}= & {\left[\begin{array}{cccc}
0 & 0 & \frac{R_{r}}{L_{o}} & \frac{\beta_{2} \omega_{r}}{L_{o}} \\
0 & 0 & -\frac{\hat{\beta}_{2} \omega_{r}}{L_{o}} & \frac{R_{r}}{L_{o}} \\
\frac{M R_{r}}{L_{r}} & 0 & 0 & -\frac{\beta_{2} \omega_{r}}{L_{r}} \\
0 & \frac{M R_{r}}{L_{r}} & \frac{\beta_{2} \omega_{r}}{L_{r}} & 0
\end{array}\right], \mathrm{B}=\left[\begin{array}{cc}
\frac{\beta_{3}}{L_{o}} & 0 \\
0 & \frac{\beta_{3}}{L_{o}} \\
0 & 0 \\
0 & 0
\end{array}\right] }
\end{aligned}
$$

Note that the matrix $A_{0}$ is a negative-definite matrix.

\section{Theorem 1.}

Consider an induction motor whose dynamics is governed by (1) under assumptions (A1) to (A6) If the stator current observers and the rotor flux observers are designed as in (6), then the mechanical angular speed of the rotor $\omega_{r}$ will be driven to approach to a bounded smooth speed command $\omega_{r d}$ by the following control law

$$
u_{1}=\frac{L_{o}}{\beta_{3}}\left[-\frac{\hat{R}_{r}}{L_{o}} \hat{\psi}_{a}-\frac{\beta_{2}}{L_{o}} \hat{\omega}_{r} \hat{\psi}_{b}+\dot{I}_{a d}\right.
$$




$$
\begin{aligned}
& \left.+\left(\frac{M}{L_{o}} \hat{R}_{r}+\frac{\beta_{1}}{L_{o}}\right) I_{a d}-k_{1} e_{1}+v_{e 11}+v_{e 12}\right] \\
u_{2}= & \frac{L_{o}}{\beta_{3}}\left[-\frac{\hat{R}_{r}}{L_{o}} \hat{\psi}_{b}+\frac{\beta_{2}}{L_{o}} \hat{\omega}_{r} \hat{\psi}_{a}+\dot{I}_{b d}\right. \\
& \left.+\left(\frac{M}{L_{o}} \hat{R}_{r}+\frac{\beta_{1}}{L_{o}}\right) I_{b d}-k_{1} e_{2}+v_{e 21}+v_{e 22}\right]
\end{aligned}
$$

with the desired signals being designed as

$$
\begin{aligned}
T_{d} & =\left(J+\mu_{2}\right) \dot{\omega}_{r d}+\left(D+\mu_{1}\right) \omega_{r d}+\mu_{0} \cdot s\left(k, \omega_{r d}\right) \\
\dot{\psi}_{a d} & =-\left(\frac{\beta_{2}}{L_{r}} \hat{\omega}_{r}+\frac{M T_{d}}{\gamma^{2} L_{r} k_{T}} \hat{R}_{r}\right) \psi_{b d} \\
\dot{\psi}_{b d} & =\left(\frac{\beta_{2}}{L_{r}} \hat{\omega}_{r}+\frac{M T_{d}}{\gamma^{2} L_{r} k_{T}} \hat{R}_{r}\right) \psi_{a d} \\
I_{a d} & =-\frac{T_{d}}{\gamma^{2} k_{T}} \psi_{b d}+\frac{1}{M} \psi_{a d} \\
I_{b d} & =-\frac{T_{d}}{\gamma^{2} k_{T}} \psi_{a d}+\frac{1}{M} \psi_{b d}
\end{aligned}
$$

for known positive constants $\mu_{0}, \mu_{1}$ and $\mu_{2}$, auxiliary control laws :

$$
\begin{aligned}
u_{o 1}= & -\frac{\hat{R}_{r}}{L_{r}}\left(L_{o} \tilde{I}_{a}-\zeta_{a}\right)-\frac{\beta_{2} \hat{\omega}_{r}}{L_{r}}\left(L_{o} \tilde{I}_{b}-\zeta_{b}\right) \\
u_{o 2}= & -\frac{\hat{R}_{r}}{L_{r}}\left(L_{o} \tilde{I}_{b}-\zeta_{b}\right)+\frac{\beta_{2} \hat{\omega}_{r}}{L_{r}}\left(L_{o} \tilde{I}_{a}-\zeta_{a}\right) \\
u_{o 3}= & -k_{0} \tilde{I}_{a}-u_{o 1}-u_{c 1} \\
u_{o 4}= & -k_{0} \tilde{I}_{b}-u_{o 2}-u_{c 2} \\
u_{c 1}= & \frac{1}{L_{r}} \operatorname{sgn}\left(\tilde{I}_{a}\right) \cdot\left(F_{1}(t)+\beta_{2} F_{2}(t)\right) \\
u_{c 2}= & \frac{1}{L_{r}} \operatorname{sgn}\left(\tilde{I}_{b}\right) \cdot\left(F_{3}(t)+\beta_{2} F_{4}(t)\right) \\
v_{e 11}= & \frac{1}{L_{r}} \hat{R}_{r}\left(L_{o} \tilde{I}_{a}-\zeta_{a}\right)+\frac{\beta_{2}}{L_{r}} \hat{\omega}_{r}\left(L_{o} \tilde{I}_{b}-\zeta_{b}\right) \\
v_{e 21}= & \frac{1}{L_{r}} \hat{R}_{r}\left(L_{o} \tilde{I}_{b}-\zeta_{b}\right)-\frac{\beta_{2}}{L_{o} L_{r}} \hat{\omega}_{r}\left(L_{o} \tilde{I}_{a}-\zeta_{a}\right) \\
v_{e 12}= & -g_{1} \frac{1}{L_{o} L_{r}} \operatorname{sgn}\left(e_{1}\right) \cdot\left(F_{1}(t)+\beta_{2} F_{2}(t)\right) \\
v_{e 22}= & -g_{1} \frac{1}{L_{o} L_{r}} \operatorname{sgn}\left(e_{2}\right) \cdot\left(F_{3}(t)+\beta_{2} F_{4}(t)\right) \\
\dot{\zeta}_{a}= & -\frac{1}{k_{\eta}}\left[-\frac{1}{L_{r}} \hat{R}_{r} \tilde{I}_{a}+\frac{\beta_{2}}{L_{r}} \hat{\omega}_{r} \tilde{I}_{b}-\frac{g_{1}}{L_{o} L_{r}} \hat{R}_{r} e_{1}\right. \\
& \left.+g_{1} \frac{\beta_{2}}{L_{o} L_{r}} \hat{\omega}_{r} e_{2}\right] \\
\dot{\zeta}_{b}= & -\frac{1}{k_{\eta}}\left[-\frac{1}{L_{r}} \hat{R}_{r} \tilde{I}_{b}-\frac{\beta_{2}}{L_{r}} \hat{\omega}_{r} \tilde{I}_{a}-\frac{g_{1}}{L_{o} L_{r}} \hat{R}_{r} e_{2}\right. \\
& \left.-g_{2} \hat{\omega}_{r} e_{1}\right] \\
L_{r} &
\end{aligned}
$$

where

$$
\begin{array}{ll}
F_{1}(t) \geq \delta_{a}\left|\tilde{R}_{r}\right|, & F_{2}(t) \geq \delta_{b}\left|\tilde{\omega}_{r}\right|, \\
F_{3}(t) \geq \delta_{b}\left|\tilde{R}_{r}\right|, & F_{4}(t) \geq \delta_{a}\left|\tilde{\omega}_{r}\right|
\end{array}
$$

and the rotor speed observer and the rotor resistance observer

$$
\begin{aligned}
\dot{\hat{R}}_{r}= & \frac{1}{k_{R}}\left[\Omega_{o 1}+\Omega_{c 1}+\hat{\eta}_{a}\left(\frac{1}{L_{r}} \tilde{I}_{a}+\frac{g_{1}}{L_{o} L_{r}} e_{1}\right)\right. \\
& \left.+\hat{\eta}_{b}\left(\frac{1}{L_{r}} \tilde{I}_{b}+\frac{g_{1}}{L_{o} L_{r}} e_{2}\right)+u_{R}\right] \\
\dot{\hat{\omega}}_{r}= & \frac{1}{k_{\omega}}\left[\Omega_{02}+\Omega_{c 2}-\hat{\eta}_{a}\left(\frac{\beta_{2}}{L_{r}} \tilde{I}_{b}+g_{1} \frac{\beta_{2}}{L_{o} L_{r}} e_{2}\right)\right. \\
& \left.+\hat{\eta}_{b}\left(\frac{\beta_{2}}{L_{r}} \tilde{I}_{a}+g_{1} \frac{\beta_{2}}{L_{o} L_{r}} e_{1}\right)+u_{\omega}\right]
\end{aligned}
$$

with projection laws

$$
\begin{aligned}
& u_{R}=\left\{\begin{array}{cc}
\frac{1}{2} g_{2} a^{2} \gamma^{2}\left(\underline{R}_{r}-\hat{R}_{r}\right) & \text { if } \hat{R}_{r}>\bar{R}_{r}+\delta_{R} \\
\Omega_{R}+\delta_{R 2} & \text { if } \hat{R}_{r} \leq \bar{R}_{r}+\delta_{R}
\end{array}\right. \\
& u_{\omega}=\left\{\begin{array}{cc}
\frac{1}{2} g_{2} p^{2} \gamma^{2}\left(\omega_{r d}-\delta_{\omega}-\hat{\omega}_{r}\right) & \text { if } \hat{\omega}_{r}>\omega_{r d}+\delta_{\omega} \\
\Omega_{\omega}+\delta_{\omega 2} & \text { if } \hat{\omega}_{r} \leq \omega_{r d}+\delta_{\omega}
\end{array}\right.
\end{aligned}
$$

for some constants

$$
\delta_{R}, \delta_{\omega}, \delta_{R 2}, \delta_{\omega 2}>0
$$

where

$$
\begin{aligned}
\Omega_{R}= & -\Omega_{o 1}-\Omega_{c 1}-\hat{\eta}_{a}\left(\frac{1}{L_{r}} \tilde{I}_{a}+\frac{g_{1}}{L_{o} L_{r}} e_{1}\right) \\
& -\hat{\eta}_{b}\left(\frac{1}{L_{r}} \tilde{I}_{b}+\frac{g_{1}}{L_{o} L_{r}} e_{2}\right) \\
\Omega_{\omega}= & -\Omega_{o 2}-\Omega_{c 2}+\hat{\eta}_{a}\left(\frac{\beta_{2}}{L_{r}} \tilde{I}_{b}+g_{1} \frac{\beta_{2}}{L_{o} L_{r}} e_{2}\right) \\
& -\hat{\eta}_{b}\left(\frac{\beta_{2}}{L_{r}} \tilde{I}_{a}+g_{1} \frac{\beta_{2}}{L_{o} L_{r}} e_{1}\right)
\end{aligned}
$$

and the initial conditions of $\hat{R}_{r}$ and $\hat{\omega}_{r}$ satisfying

$$
\begin{aligned}
& \hat{R}_{r}(0)>\bar{R}_{r}+\delta_{R}, \\
& \hat{\omega}_{r}(0)>\omega_{r d}(0)+\delta_{\omega}
\end{aligned}
$$

\section{Proof:}

Select the Lyapunov function candidate as :

$V_{o}=\frac{1}{2} L_{o} \tilde{I}_{a}^{2}+\frac{1}{2} L_{o} \tilde{I}_{b}^{2}+\frac{1}{2} k_{\eta} \eta_{a}^{2}+\frac{1}{2} k_{\eta} \eta_{b}^{2}+\frac{1}{2} k_{\omega} \tilde{\omega}_{r}^{2}+\frac{1}{2} k_{R} \tilde{R}_{r}^{2}$

$V_{c}=\frac{1}{2} e^{T} G e, G=\operatorname{diag}\left[g_{1}, g_{1}, g_{2}, g_{2}\right]$

$V=V_{o}+V_{c}$

for some constants $k_{\eta}, k_{\omega}, k_{R}, g_{1}, g_{2}>0$, then its time derivative is obviously the following form :

$$
\begin{aligned}
\dot{V} \leq & -k_{0} \tilde{I}_{a}^{2}-k_{0} \tilde{I}_{b}^{2} \\
& -g_{1}\left(\frac{M}{L_{o}} R_{r}+\frac{\beta_{1}}{L_{o}}+k_{1}-\frac{1}{2} \frac{g_{2} M^{2}}{g_{1} L_{r}^{2}} R_{r}^{2}\right)\left(e_{1}^{2}+e_{2}^{2}\right) \\
& -g_{2}\left(\frac{R_{r}}{L_{r}}-\frac{3}{2}\right)\left(e_{3}^{2}+e_{4}^{2}\right)
\end{aligned}
$$


By properly assigning positive constants $g_{1}, g_{2}$ and $k_{1}$, we can make $V$ as well as $-\dot{V}$ both become positive functions. A sufficient condition for the value of constant $k_{1}$ is

$$
k_{1}>\frac{1}{2} \frac{g_{2} M^{2}}{g_{1} L_{r}^{2}} R_{r}^{2}-\frac{M}{L_{o}} R_{r}-\frac{\beta_{1}}{L_{\circ}}
$$

According to (7) and standard Lyapunov stability theory [13], observation errors , parameter estimation error and state tracking errors are all bounded. Besides, from Corollary 2 , the desired state trajectories $I_{a d}, I_{b d}, \psi_{a d}$ and $\psi_{b d}$ are all bounded. Since the desired states and the state tracking errors are all bounded, the system states $I_{a}, I_{b}, \psi_{a}$ and $\psi_{b}$ are all bounded. Considering the definition of the auxiliary error signals $\tilde{Z}_{a}$ and $\tilde{Z}_{b}$, since the stator current observation errors $\tilde{I}_{a}$ and $\tilde{I}_{b}$ are both bounded, the rotor flux observation errors $\tilde{\psi}_{a}$ and $\bar{\psi}_{b}$ are bounded, too. So far, we know that the true system states and their observation errors are all bounded, which implies that the observed values $\hat{I}_{a}, \hat{I}_{b}$, $\hat{\psi}_{a}$ and $\hat{\psi}_{b}$ are then bounded. Recall that all auxiliary control signals $u_{o 1}$ to $u_{o 4}$ and $u_{c 1}, u_{c 2}, v_{e 11}, v_{e 12}, v_{e 21}$, $v_{e 22}$ are combinations of states, observed signals and observation errors and hence are all bounded. Moreover, from the dynamic model (1), the generated torque is a combination of system states, and therefore it is also a bounded function. Then, by assumption (A4) the rotor speed $\omega_{r}$ is bounded, which implies that $\hat{\omega}_{r}$ is bounded since $\tilde{\omega}_{r}$ is bounded. As to the rotor resistance estimator, since the true value is bounded naturally and the estimation error is proved to be bounded, the estimated value $\hat{R}_{r}$ is bounded. This means that all internal signals are bounded functions. Consequently, the observer and the controller design introduced above is indeed feasible and well defined. Follow a similar analysis as Barbalat's Lemma [14], we will obtain the result

$$
\tilde{I}_{a}, \tilde{I}_{b}, e_{1}, e_{2}, e_{3}, e_{4} \rightarrow 0, \quad \text { as } t \rightarrow \infty
$$

referring to Lemma 2 in the previous chapter together with the result above, we see that the torque tracking objective is then achieved

$$
T_{e} \rightarrow T_{d} \text { as } t \rightarrow \infty
$$

which in turn, implies that the speed tracking error converges to zero, too, i.e.,

$$
\begin{aligned}
& e_{5} \rightarrow 0 \quad \text { as } t \rightarrow \infty \\
\Rightarrow & \omega_{r} \rightarrow \omega_{r d} \quad \text { as } t \rightarrow \infty
\end{aligned}
$$

from Lemma 1. This completes the stability analysis of the overall system.

\section{Experimental Result}

Experiments are done with a three horse power induction motor which is manufactured by TECO Oo. Ltd.
Taiwan. Parameters of the motor are listed in table 4.1.

\begin{tabular}{llll}
\hline \multicolumn{2}{c}{ Specification } & & Parameter \\
\hline Poles & 4 & $R_{s}$ & $0.83 \Omega$ \\
Rated current & $8.6 \mathrm{~A}$ & $R_{r}$ & $0.53 \Omega$ \\
Rated voltage & $220 \mathrm{~V}$ & $L_{s}$ & $86.01 \mathrm{mH}$ \\
Rated frequency & $60 \mathrm{~Hz}$ & $L_{r}$ & $86.01 \mathrm{mH}$ \\
Rated Speed & $1720 \mathrm{RPM}$ & $M$ & $82.59 \mathrm{mH}$ \\
Rated power & $2.2 \mathrm{KW}$ & $J$ & $0.033 \mathrm{~kg} \cdot \mathrm{m}^{2}$ \\
& & $D$ & $0.00825 \mathrm{~N} \cdot \mathrm{m} \cdot \mathrm{s}$ \\
\hline
\end{tabular}

Table 1. Specifications and Parameters of the Motor

In order to check the performance, we have done two experiments with exponential and sinusoidal speed command as in figure 1 and figure 2 .

Obviously, experiments show that the sensorless controller is indeed effective to drive the motor to track a given smooth speed command.
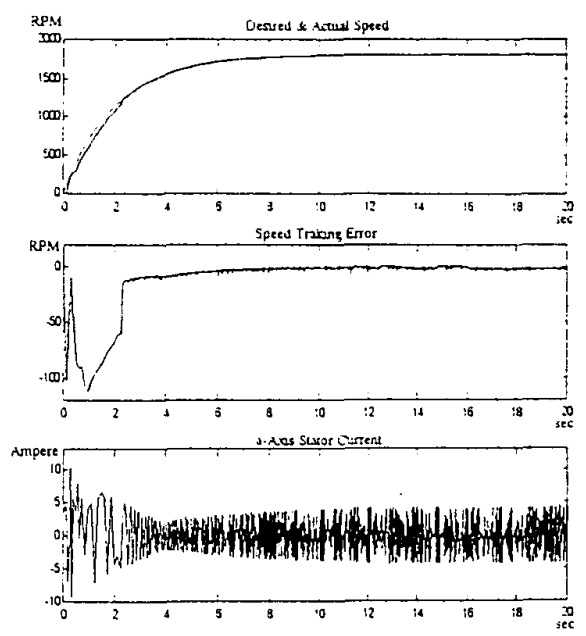

Figure 1. $\omega_{r d}=1800\left(1-e^{-0.5 t}\right)$ RPM with no load

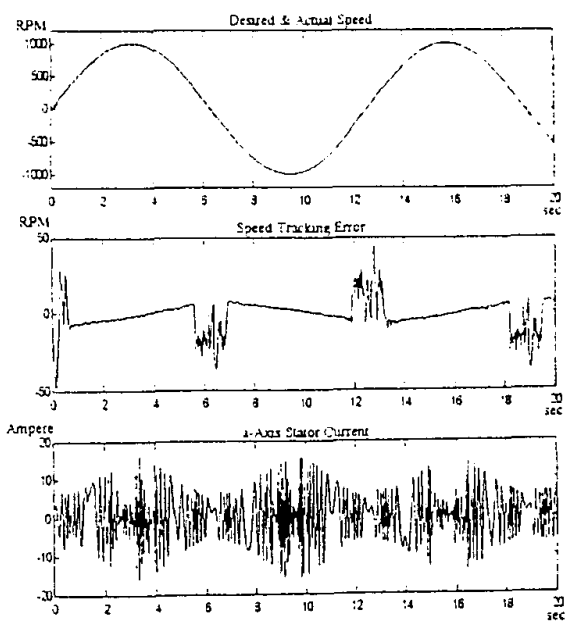

Figure 2. $\omega_{r d}=1000 \cdot \sin (0.5 t) \mathrm{RPM}$ with no load 


\section{Conclusion}

In this paper, we have presented a singularity-free, partial-state feedback adaptive sensorless speed tracking controller for induction motors with fifth-order nonlinear dynamic model which is actuated by a voltage source. The main contribution of the controller is that asymptotic rotor speed tracking is achieved without measurement of both the rotor fluxes and the rotor speed. Moreover, the variation of the rotor resistance is also taken into account. In addition to the rotor speed, we can also control the norm of the rotor fluxes to track a given constant value. However, the controller is not free of defects. The main weakness of this controller is that the rotor speed is assumed to be slowly varying and the difference between it and the desired speed command siould be less than or equal to a bound $\delta_{\omega}$.

\section{References}

[1] W. Leonhard, "Microcomputer Control of High Dynamic Performance Ac-Drives-a Survey", Automatica, Vol. 22, pp. 1-19, 1986.

[2] A. D. Luca and G. Ulivi, "Design of an Exact Nonlinear Controller for Induction Motors", IEEE Trans. on Automatic Control, Vol. 34, No. 12, pp. 1304-1307, 1989.

[3] R. Marino, S. Peresada and P. Valigi, "Adaptive Input-Output Linearizing Control of Induction Motors", IEEE Trans. on Automatic Control, Vol. 38, pp. 208-221, 1993.

[4] A. Bellini, G. Figalli and G. Ulivi, "Analysis and Design of a Microcomputer- Based Observer for an Induction Machine", Automatica, Vol. 24, pp. 549$555,1988$.

[5] G. C. Verghese and S. R. Sanders, "Observers for Flux Estimation in Induction Machines", IEEE Trans. on Industrial Electronics, Vol. 35, pp. 85-94, 1988.

[6] J. Stephan, M. Bodson and J. Chiasson, "Real-Time Estimation of the Parameters and Fluxes of Induction Motors", IEEE Trans. on Industrial Applications, Vol. 30, No. 3, pp. 746-758, 1994.

[7] R. Marino, S. Peresada and P. Tomei, "Exponentially Convergent Rotor Resistance Estimation for Induction Motors", IEEE Trans. on Industrial Electronics, Vol. 42, No. 5, pp. 508-515, 1995.

[8] J. Hu and D. M. Dawson, "Adaptive Control of Induction Motor Systems Despite Rotor Resistance Uncertainty", Proceedings of the American Control Conference, Jun. 1996, pp. 1397-1402

[9] G. Espinosa and R. Ortega, "State Observers are unnecessary for Induction Motor Control", Systems $\&$ Control Letters, Vol. 23, No. 5, 1994, pp. 315-323
[10] A. M. Lee and L. C. Fu, "Nonlinear Adaptive Speed and Torque Control of Induction Motors with Unknown Rotor Resistance", Master Thesis National Taiwan University Taiwan R.O.C., 1996.

[11] P. C. Krause, "Analysis of Electric Machinery", Book McGraw-Hill 1987.

[12] T. Ohtani, N. Takada and K. Tanaka, "Vector Control of Induction Motor without Shaft Encoder", IEEE Trans. on Industrial Application, Vol. 28, No. 1, Jan./Feb. 1992, pp. 157-164.

[13] M. Vidyasagar, "Nonlinear System Analysis", Englewood Cliffs, NJ: Prentice-Hall, 1992.

[14] K. S. Narendra, A. M. Annaswamy, "Stable Adaptive Systems", Englewood Cliffs, NJ: Prentice-Hall, 1989.

[15] H. Kubota and K. Matsuse, "Speed Sensorless Field-Oriented Control of Induction Motor with Rotor Resistance Adaptation", IEEE Trans. on Industrial Application, Vol. 30, No. 5, Sep./Oct. 1994, pp. 1219-1224.

[16] Jung-Hua Yang, Wen-Hai Yu, and Li-Chen Fu, "Nonlinear Observer-Based Adaptive Tracking Control for Induction Motors with Unknown Load", IEEE Trans on Industrial Electronics, Vol. 42, No. 6, Dec. 1995, pp. 579-586.

[17] C. Has, A. Bettini, L. Feraris, G. Griva and F. Profumo, "Comparison of Different Schemes without Shaft Sensors for Field Oriented Control Drives", Proceedings of IEEE IECON'94, pp. 1579-1588, 1994.

[18] T. H. Chin, "Approaches for Vector Control of Induction Motor without Speed Sensors", Proceedings of IEEE IECON'94, pp. 1616-1620, 1994.

[19] R. Marino, S. Peresada and P. Tomei, "Adaptive Observer-Based Control of Induction Motors with Unknown Rotor Resistance", IEEE International Journal of Adaptive Control and Signal Processing, Vol. 10, 1996, pp.345-363.

[20] R. Marino, S. Peresada and P. Tomei, "Global Adaptive Output Feedback Control of Induction Motors with Uncertain Rotor Resistance", Proceedings of the 35th Conference on Decision and Control, pp. 4701-4706, 1996. 